\title{
ANALYSIS OF SPECTROSCOPIC OBSERVATIONS OF JUPITER AND THE VARIABILITY OF THE STRUCTURE OF THE VISIBLE CLOUDS
}

\author{
G. E. HUNT \\ Meteorological Office, Bracknell, Berkshire, England \\ and \\ J. T. BERGSTRALH \\ Jet Propulsion Laboratory, Pasadena, Calif., U.S.A.
}

\begin{abstract}
Realistic inhomogeneous models of Jupiter's lower atmosphere are used to interpret recent observations of the (3-0) and (4-0) $\mathrm{H}_{2}$ quadrupole lines at different portions of the visible disc of the planet. The time variations of these observations are analysed in terms of changes in the cloud structure.
\end{abstract}

\section{Introduction}

In an earlier publication, Bergstralh (1973) reported a quantitative "mapping" of the strengths of the $\mathrm{CH}_{4} 3 v_{3} R$-branch manifolds across the Jovian disk, and concluded from the behaviour of these lines that a layered inhomogeneous scattering model for absorption line formation was the most satisfactory of three considered. We conducted a similar mapping program during the 1972 apparition of Jupiter, this time in the $S(1)$ lines of the $\mathrm{H}_{2}(3-0)$ and (4-0) quadrupole bands, since an analysis by Hunt (1973a) indicated that the $\mathrm{H}_{2}$ line strengths were more sensitive than the $\mathrm{CH}_{4}$ lines to conditions of line formation. To ensure that variations of $\mathbf{H}_{2}$ absorption with time and/or zenographic longitude did not introduce spurious results, we also instituted a 'patrol' of the $\mathrm{H}_{2}$ line strengths at the centre of the Jovian disk.

\section{Observations}

Observations were obtained with the coudé spectrographs of the $208 \mathrm{~cm}$ (82 in.) Struve telescope at McDonald Observatory and the $61 \mathrm{~cm}$ (24 in.) telescope at Jet Propulsion Laboratory's Table Mountain Observatory. Because of its large image scale, we used the McDonald telescope for spatial mapping of the line strengths. The Table Mountain telescope was used for the central meridian patrol since its image scale was adequate for the purpose, and large blocks of its time could be committed to the program. In addition, a very limited amount of spatial mapping was attempted at Table Mountain.

All spectra were recorded on photographic plates, using a two-stage S-1 image intensifier tube at McDonald and a single-stage S-25 tube at Table Mountain. Spectra were obtained at $1.6 \AA \mathrm{mm}^{-1}$ reciprocal dispersion in the (4-0) band and $2 \AA \mathrm{mm}^{-1}$ in the 
(3-0) band at both observatories. The plates were traced with a digital microphotometer at JPL, and the digital data reduced to intensity tracings by a computer routine using conventional techniques of photographic photometry.

All of the spectra reported here were obtained with the image of Jupiter held in a pole-to-pole orientation along the spectrograph slit by means of an image rotator. The scale of the McDonald spectra allowed them to be traced in three strips parallel to the dispersion; a central strip corresponding to the dusky equatorial zone, between about $\pm 15^{\circ}$ zenographic latitude, and strips on either side corresponding to the brighter north and south tropical zones, between about $\pm 15^{\circ}$ and $\pm 49^{\circ}$ latitude. The Table Mountain image scale was such that a rather broad strip, between about $\pm 30^{\circ}$ latitude, was the narrowest region that could be traced with signal-to-noise adequate for quantitative work.

To check the photometric calibration, the equivalent widths of several Fraunhofer lines, selected to match approximately the strengths and wavelengths of the Jovian $\mathrm{H}_{2}$ lines, were measured on each tracing. The measured strengths of the former were systematically low on the tracings of the Table Mountain spectra and systematically a bit high on the McDonald tracings; linear least-squares fits of 'measured' to 'known' line strengths were therefore computed to correct the measurements of the $\mathrm{H}_{2}$ lines. Error estimates given with individual measurements reported here are standard $(1 \sigma)$ errors computed from the least-squares fits.

When the Table Mountain measurements of (3-0) and (4-0) S(1) were plotted against date of observation, the points appeared to be randomly distributed, with external variances approximately equal to the estimated errors of individual measurements. Indeed, the apparent variations of the line strengths were not significant at the $5 \%$ level by the $F$-test. However, when the same measurements were plotted against System I longitude, a broad pattern emerged; the strengths of the (3-0) and (4-0) S (1) lines appeared to decrease by about 10 to 15 per cent between $220^{\circ}$ and $280^{\circ}$ longitude. Evidently, real variations of small amplitude occurred in the hydrogen line strengths. We are attempting now to correlate these apparent variations with visible features of the Jovian cloud deck, photographed at about the time of our observations.

\section{Atmospheric Model}

The measurements have been interpreted by constructing a realistic model atmosphere of Jupiter in which all the processes of radiative transfer have been accurately taken into account. The details are described in Hunt (1973b). Hydrogen quadrupole lines exhibit the phenomenon of collision narrowing before the more usual pressure broadening takes place and it is important that the current line shape is used in an analysis, Hunt and Margolis (1973). We have used the Galatry line shape which accurately represents the quadrupole line shape. The nominal model of the Jovian atmosphere proposed by Divine (1971), which is consistent with our present knowledge, is used as a basis of this analysis. 


\section{Results}

At the equatorial region of Jupiter a structure of two distinct cloud layers is consistent with all observations. Hunt (1937b) showed the upper cloud layer to be optically thin. In the analysis of these observations we find it has negligible effect upon the equivalent widths observed at the centre of the disc. Consequently observations of this type may be interpreted by a reflecting layer technique. Then the changes in the derived effective pressure at the level of line formation of weak lines, such as the (4-0) S(1), will indicate the variability in the top of the dense, lower cloud.

The observations carried out during June 1972 show a decrease in all measured line strengths for System I longitude in the range 200 to $280^{\circ}$. For the (4-0) S(1) line this requires the effect pressure at the level of line formation to vary from $\sim 2 \pm 0.5$ to $\sim 1.25 \pm 0.2 \mathrm{~atm}$ and the abundance from $\sim 68 \pm 15$ to $89 \pm 13 \mathrm{~km}$ amagat. For the (3-0) S(1) line, a saturated and therefore less sensitive line, the pressure varies from $\sim 1.6 \pm 0.5$ to $\sim 1 \pm 0.4 \mathrm{~atm}$ and the abundance from $\sim 54 \pm 16$ to $\sim 33 \pm 13 \mathrm{~km}$ amagat. This requires the lower cloud to rise by $\sim 1$ scale height during the observational period from the $\sim 2$ atm level.

During August 1972 the observations indicated an increase in line strength in all measured lines over the System I longitude range of 180 to $320^{\circ}$. For the (4-0) S(1) line this requires the effective pressure to increase from $1.88 \pm 0.37$ to $4.43 \pm 0.93 \mathrm{~atm}$; the abundance from $61.25 \pm 11.75$ to $145 \pm 30 \mathrm{~km}$ amagat. The corresponding change for the (3-0) $\mathrm{S}(1)$ line is $1.18 \pm 0.52$ to $2.26 \pm 0.89$ atm and $38.25 \pm 16.75$ to $73.75 \pm 29.25 \mathrm{~km}$ amagat. This requires the level of the lower cloud to decrease by more a scale height during the observational period of two Earth days from the 1.88 atm level.

\section{Conclusion}

The patrol of $\mathrm{H}_{2}$ quadrupole line strengths at the centre of the Jovian disk during 1972 apparition indicate great variability in the measured equivalent widths. This has been shown to be correlated with changes in the level of the lower cloud which behaves as a reflecting surface.

\section{References}

Bergstralh, J. T.: 1973, Icarus 19, 390.

Divine, N.: 1971, 'The Planet Jupiter' SP, 8069.

Hunt, G. E.: 1973a, Icarus 18, 637.

Hunt, G. E.: 1973b, Monthly Notices Roy. Astron. Soc. 161, 347.

Hunt, G. E. and Margolis, J. S.: 1973, J. Quant. Spectrosc. Radiat. Transfer 13, 417.

\section{DISCUSSION}

Gulkis: What is the significance of plotting the data in System I longitude?

Owen: The data were not only restricted to the central meridian longitude but also to the equatorial region. 